\title{
Searches for Exotic Long lived Particles using Early Data from the ATLAS Detector at the LHC
}

\author{
Fabrizio Salvatore* \\ (on behalf of the ATLAS Collaboration) \\ University of Sussex \\ E-mail: p.f.salvatorelsussex.ac.uk
}

\begin{abstract}
R-hadrons are massive long-lived particles (LLPs) which are featured in a wide range of different Supersymmetric models. In this paper we summarize the strategies for the search for LLPs in the ATLAS detector using Time-of-Flight techiniques. Moreover, we describe a first search for gluino R-hadrons which have come to rest within the ATLAS detector and decay some time later. Candidate events are selected in the time interval between two consecutive bunch crossings of the LHC in order to remove collision backgrounds. Simple selection criteria can be defined in order to discriminate between signal-like events and backgrounds, the largest of which is due to cosmic muons. The event yield from data collected during collisions at $\sqrt{s}=7 \mathrm{TeV}$ is found to be in good agreement with the yields in the 2009 cosmic data sample.
\end{abstract}

35th International Conference of High Energy Physics - ICHEP2010,

July 22-28, 2010

Paris France

\footnotetext{
${ }^{*}$ Speaker.
} 


\section{Introduction}

The search for a new type of heavy long-lived particle (LLP) is an important component of the early data exploitation program of the LHC $[1,2]$. LLPs containing a heavy colored particle are called R-hadrons. They are predicted by many theories beyond the Standard Model (SM) [3, 4, 5], which postulate the existence of non-SM particles with masses of the order of the TeV to solve the so-called gauge hierarchy problem. Since these particles can be relatively slow $(\beta \ll 1)$, a promising way to detect them in the ATLAS detector [6] is to use time-of-flight (ToF) measurements. The mass of the candidate LLP could be obtained by exploiting the timing information of the Tile Calorimeter (TileCal) and Muon Spectrometer (MS) to calculate the particle's velocity, and combine this with the measurement of the momentum $p$ of the LLP $(m=p / \beta \gamma)$. The strategy in ATLAS for this analysis and the timing performance of the TileCal obtained from single beam events collected at the start of the 2010 run are being described. At the LHC energies, gluino R-hadrons would be produced in pairs, approximately back-to-back in the transverse plane. Some fraction of them can loose all their momenta and come to rest within the detector volume ("stopped" gluinos), where they then decay at some later time. The first studies to discriminate signal-like events from backgrounds, the largest of which come from cosmic ray interactions, will be described.

\section{Strategy for LLP searches using time-of-flight technique in the ATLAS Tile Calorimeter and Muon Spectrometer}

The Tile Calorimeter constitues the barrel part of the ATLAS hadronic calorimetry. A full description of this detector, as well as of all the ATLAS detectors, can be found in [6]. The central barrel $(|\eta|<0.8)$ is divided in two partitions (Long Barrel A and B), with two Extended Barrel Regions (A and B) covering the region $0.8 \leq|\eta| \leq 1.7$. Each partition is segmented in $\phi$ into 64 modules, which are divided in cells that are grouped radially in three layers $(\mathrm{A}, \mathrm{BC}$ and D). The selection of LLP candidates in the ToF analysis in the TileCal starts with a muon candidate reconstructed in the Muon Spectrometer (MS), as well as inner detector tracks that are tagged as muon by their characteristic energy deposit in the Tile calorimeter. The speed $\beta$ of an LLP candidate can then be measured using the time measurement of the TileCal cell in which it deposited its energy. If several cells along the extrapolated track of the reconstructed candidate are taken into account, the speed can be calculated as $\beta_{\text {Tile }}=\frac{\sum_{i=0}^{n} w_{i} \beta_{i}}{\sum_{i=0}^{n} w_{i}}$. The parameter $w_{i}\left(E_{i}\right)=E_{i}$ is the weight for the measurement of $\beta$ in cell $i$, and $n$ is the number of cells along the reconstructed path where the energy deposted has exceeded a given threshold. Given the analysis strategy for these searches, in order to be able to perform a ToF analysis using the TileCal detector it is important to understand its timing and energy calibration. The timing TileCal signals recorded with single beam data during February 2010 are shown in Figure 1 (left) [7]. The average time over all cells with the same $\phi$ coordinate is shown in function of the cell $z$-coordinate (along the beam axis), for all three radial samplings. The observed slopes match the time the muons take to cross the calorimeter along the $z$-direction. In Figure 1 (right) the time of flight correction that assumed a track parallel to the $z$-axis is applied, obtaining a flat distribution (within $1 \mathrm{ns)} \mathrm{which} \mathrm{demonstrates} \mathrm{a} \mathrm{very} \mathrm{good}$ time equalization. 

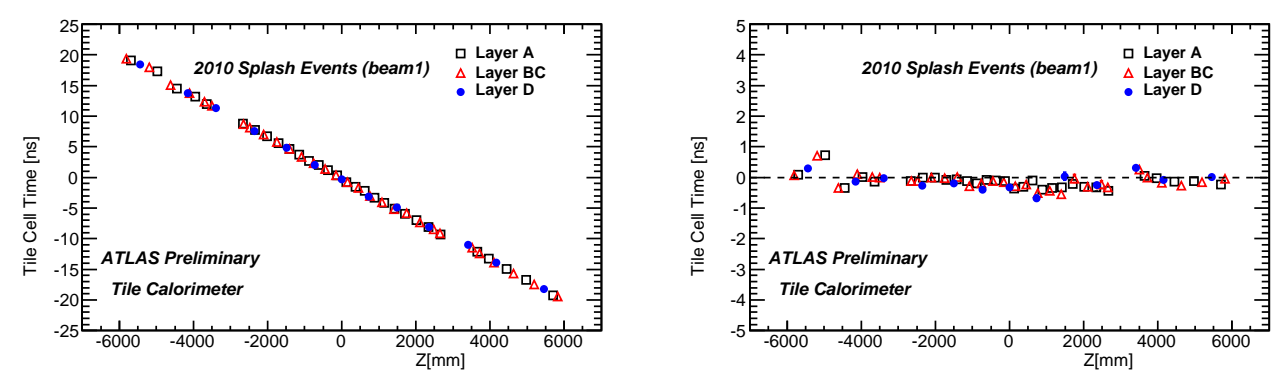

Figure 1: Timing of TileCal signals recorded with single beam data on February 2010. Left: observed slopes match the time the muons take to cross the calorimeter along the $z$-direction. Right: distribution obtained after applying the time of flight correction that assumed a track parallel to the $z$-axis.

The speed of slow particles could also be estimated using muon candidates reconstructed by the MS only. The reconstruction is based on recovering trigger detector hits from the next bunchcrossing, estimating the particle velocity from the Resistive Plate Chambers (RPC) hit time and selecting the the $\beta$ that minimizes the segments $\chi^{2}$ in the precision muon chambers [8]. The efficiency reconstructing $\beta$ is over $90 \%$ for simulated candidates with $\beta>0.4$.

\section{Stopped Gluino Searches in ATLAS}

The analysis performed to search for stopped gluino candidates in ATLAS [10] relies mainly on the information from the Liquid Argon (LAr) sampling calorimeter, TileCal and the muon system [6]. Jet triggers obtained from the calorimeter [9], which fire during the empty bunch crossing of the LHC running at $\sqrt{s}=7 \mathrm{TeV}$, are used to select the candidate events. The criteria to isolate signal-like events from backgrounds is to select events with less than 4 jets and no tracks or segments reconstructed in the muon system. The leading jet should have an energy of at least $50 \mathrm{GeV}$ and be central in $\eta(|\eta|<1.2)$. Further requirements are applied to remove noisy calorimeter cells. The events expected in such sample come primarily from cosmic rays, with some negligible residual beam-halo and beam-gas background. In order to test this assumption, the collision data collected between March and June 2010 (corresponding to $2.7 \pm 0.3 \mathrm{nb}^{-1}$ ) have been compared to cosmic ray events collected during the commissioning of the experiment between May and November 2009, where the detector configuration and reconstruction algorithms matched closely those of the collision data taking. The cosmic ray sample corresponds to $5.42 \times 10^{6}$ events satisfying the requirement of having a $10 \mathrm{GeV}$ Jet at Level-1 trigger. Figure 2 shows good agreement between the distribution of the jet energy from the collision data sample and cosmic ray events. The number of background events in the cosmic ray and collision data sample is summarized in Table 3. For the purpose of the preliminary studies, we can therefore conclude that the empty bunch triggered data collected during collisions at $\sqrt{s}=7 \mathrm{TeV}$ is in good agreement with the yields in the 2009 cosmic data sample.

\section{References}

[1] The ATLAS Collaboration, G.Aad et al., "Expected Performance of the ATLAS Experiment Detector, Trigger and Physics”, arXiv:0901.0512 [hep-ex]. 

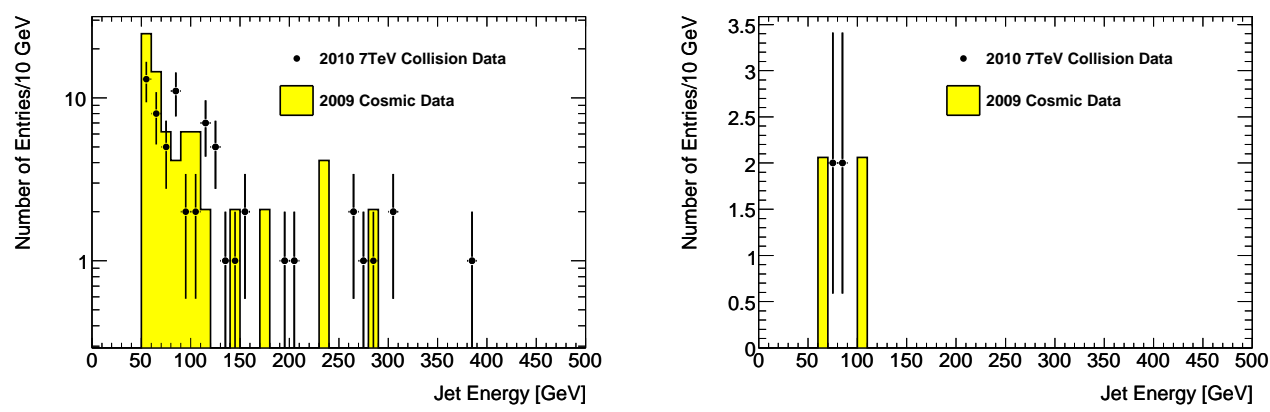

Figure 2: Jet energy for empty bunch triggers in $\sqrt{s}=7 \mathrm{TeV}$ collisin data (dots) compared with 2009 cosmic ray data (histogram). Left: leading jet energy before jet energy cut. Right: leading jet energy after all cuts.

\begin{tabular}{|l|c|c|c|}
\hline & \multicolumn{2}{|c|}{2009 Cosmic Data } & 2010 Collision Data \\
\hline Selection Criteria & Yield of cosmics & Cosmics (scaled) & Yield of data \\
\hline \hline Good runs and data quality cuts & $9.43 \times 10^{5}$ & - & $1.58 \times 10^{6}$ \\
Leading Jet $|\eta|<1.2$ & $6.26 \times 10^{5}$ & $1.29 \times 10^{6}$ & $1.29 \times 10^{6}$ \\
Jet n90 $>3$ & $3.83 \times 10^{5}$ & $7.89 \times 10^{5}$ & $7.90 \times 10^{5}$ \\
number of Jets $<4$ & $3.82 \times 10^{5}$ & $7.87 \times 10^{5}$ & $7.83 \times 10^{5}$ \\
Muon Segment Veto & $530 \pm 23.0$ & $1092 \pm 47.4$ & 1170 \\
Leading Jet Energy $>50 \mathrm{GeV}$ & $39 \pm 6.2$ & $80 \pm 12.8$ & 75 \\
Leading Jet Width $>0.05$ & $6 \pm 2.4$ & $12 \pm 4.9$ & 8 \\
Jet n50 $<6$ & $3 \pm 1.7$ & $6 \pm 3.5$ & 4 \\
Leading Jet EMF $<0.95$ & $2 \pm 1.4$ & $4 \pm 2.9$ & 4 \\
\hline \hline
\end{tabular}

Table 1: Selection criteria overview table for 2009 cosmic data collected between May and November 2009 compared with the 2010 collision data collected between March and June 2010.

[2] The CMS Collaboration, CMS Analysis Note: PAS EXO-08-003 (2008).

[3] N.Arkani-Hamed, S.Dimopoulos, G.F.Giudice and A.Romanino, "Aspects of split supersymmetry", Nucl.Phys.B 709 (2005) 346.

[4] S.Rabi, “Gauge-ediated SUSY breaking at an intermediate scale”, Phys.Rev.D 56 (1997) 2852.

[5] T.Appelquist, H.Cheng and B.A.Dobrescu, "Bounds on universal extra dimensions", Phys.Rev.D 64 (2001).

[6] The ATLAS Collaboration, G.Aad et al., "The ATLAS Experiment at the CERN Large Hadron Collider", JINST 3 (2008) S08003.

[7] The ATLAS Collaboration, G.Aad et al., "Readinessof the ATLAS Tile Calorimeter for LHC Collisions", arXiv:1007.5423v1 [physics.ins-det] (submitted to European Physics Journal C).

[8] S.Tarem, S.Bressler, H.Nomoto and A.Di Mattia, "Trigger and reconstruction for heavy long-lived charged particles with the ATLAS detector”, Eur.Phys,J. C 62 (2009) 281-292.

[9] The ATLAS Collaboration, G.Aad et al., "Properties of Jets and Inputs to Jet Reconstruction and Calibration with the ATLAS Detector using Proton-Proton Collisions at $\sqrt{s}=7 \mathrm{TeV}$, ATLAS-CONF-2010-053.

[10] The ATLAS Collaboration, G.Aad et al., "Background studies to searches for long-lived stopped particles, decaying out-of-time with the LHC collisions", ATLAS-CONF-2010-071. 